\title{
La unidad de la sustancia inmaterial en Ralph Cudworth
}

\author{
Unity in Ralph Cudworth's immaterial substance
}

Natalia Strok

\begin{abstract}
Resumen: En este artículo me propongo mostrar que en el universo armónico que propone Ralph Cudworth (1617-1688), destacado miembro del grupo de los Platónicos de Cambridge del siglo XVII, la sustancia inmaterial cumple un rol fundamental. Ella no se halla separada de la sustancia material y recibe distintos nombres de acuerdo a las funciones que desarrolla, en tanto es la única fuerza vital en la creación, fundada en la naturaleza divina. Es decir, la naturaleza plástica (plastic nature) y las almas no son más que distintos nombres para esa única sustancia inmaterial en el universo creado. Esto refleja una concepción dualista diferente al dualismo cartesiano, que tendrá consecuencias a nivel físico. Por eso, en primer lugar, presentaré la metafísica de Cudworth, para luego detenerme en la sustancia inmaterial. Para esto utilizaré su obra principal The True Intellectual System of the Universe (1678) y sus dos escritos de publicación póstuma: Treatise concerning eternal and immutable Morality (1731) y Treatise on Freewill (1838).
\end{abstract}

Palabras clave: Ralph Cudworth, Dualismo, Naturaleza plástica, Alma, Sustancia inmaterial.

Abstract: In this paper I intend to show that the immaterial substance has a fundamental role in the harmonic universe of Ralph Cudworth (1617-1688), noted member of the Cambridge Platonists group of the $17^{\text {th }}$ Century. This substance is not separated from the material one, and receives different names according to its functions, as it is the only vital force in creation, founded in the divine nature. This means that plastic nature and souls are only different names for the only immaterial substance in the created universe. This is another kind of dualism than the cartesian one, which shows consequences in a physical level. In order to do this, first, I will

\footnotetext{
* Doctora en Filosofía por la Universidad de Buenos Aires e Investigadora Adjunta del Consejo Nacional de Investigaciones Científicas y Técnicas (CONICET). Es Jefa de Trabajos Prácticos de la cátedra de Historia de la Filosofía Medieval de la Facultad de Filosofía y Letras de la Universidad de Buenos Aires, y Ayudante en la cátedra de Metafísica en la Facultad de Humanidades y Ciencias de la Educación de la Universidad Nacional de La Plata. Dirección electrónica:natiska@gmail.com
} 
present Cudworth's metaphysics, and then I will concentrate on the immaterial substance. For this I will use his main work The True Intellectual System of the Universe (1678) and his posthumous writings: Treatise concerning eternal and immutable Morality (1731) y Treatise on Freewill (1838).

Key words: Ralph Cudworth, Dualism, Plastic nature, Soul, Immaterial substance.

Ralph Cudworth es un filósofo del siglo XVII que es incluido en el grupo de los Platónicos de Cambridge. Su fuerte conexión con la universidad no evitó que participara de la vida política en la vertiginosa Inglaterra de su tiempo. ${ }^{1} \mathrm{Su}$ obra principal es The True Intellectual System of the Universe (1678), ${ }^{2}$ extenso escrito que es una primera parte del proyecto amplio que el autor se había planteado, aunque ella presente una unidad cierta en sí misma. Fue traducida al latín por Johan Lorenz Mosheim en 1733 y tuvo circulación en Europa. ${ }^{3}$ Además, Cudworth dejó una cantidad no despreciable de manuscritos, entre los cuales se encontraban sus dos obras de publicación póstuma: Treatise concerning Eternal and Immutable Morality (1731) y Treatise on Freewill (1838). ${ }^{4}$

${ }^{1}$ Carter, Benjamin, "The Standing of Ralph Cudworth As a Philosopher", en G.A.J. Rogers, Tom Sorell y Jill Kraye (eds.), Insiders and outsiders in seventeenth-century philosophy, New York, Taylor \& Francis, 2010, pp. 99-121.

2 De ahora en más “TIS". Las traducciones al español son propias. Las referencias corresponden a la primera edición: Cudworth, Ralph, The True Intellectual System of the Universe, Londres, Richard Royston, 1678. Se indica capítulo del libro, sección (cuando hubiera) y página.

${ }^{3}$ Sobre la suerte de Cudworth en su tiempo, ver Strok, Natalia, "El enigma de Ralph Cudworth en la historia de la filosofía", en Revista Anales del Seminario de Historia de la Filosofía, 35 (2), 2018, pp. 358-371 [357-373].

4 De ahora en más TEIM y TFW respectivamente. Las páginas de referencia corresponden a la edición de Hutton que contiene ambas obras: Cudworth, Ralph, $A$ Treatise Concerning Eternal and Immutable Morality: With A Treatise of Freewill, editado por Sarah Hutton, Cambridge: Cambridge University, 1996. En estos casos también son propias las traducciones al español. Para un panorama acerca de los manuscritos de Cudworth, ver Burden, Mark, "Ralph Cudworth's Freewill Manuscripts: A Roadmap to Dating and Analysis” en https://cprg.hypotheses.org/815, 2019; también Carter, Benjamin, The Little Commonwealth of Man: The Trinitarian Origins of the Ethical and Political Philosophy of Ralph Cudworth, Leuven, Peeters, 2011, [pp. 161-168] y Passmore, J. A., 
En este artículo me propongo mostrar que el universo armónico que plantea Cudworth presenta su unidad en la sustancia inmaterial, fundada en la naturaleza divina. Ella no se encuentra separada de la sustancia material, y propongo entender que recibe distintos nombres de acuerdo con las funciones que desarrolla, en tanto es la única fuerza vital en la creación. Es decir, la naturaleza plástica y las almas no son más que distintos nombres para esa única sustancia en el universo creado. Las características propias de esa sustancia tendrán consecuencias en la creación. Para esto daré cuenta de su metafísica dualista en la primera sección, para luego concentrarme en la sustancia inmaterial en la segunda, atendiendo a sus distinciones.

\section{El dualismo}

En el Prefacio de su obra principal, TIS, Cudworth es explícito en cuanto a su metafísica. Allí afirma que encontramos en el universo sólo dos tipos de sustancia:

Sin embargo, aquí presentamos toda la escala de entes y (son) considerados los rangos generales de seres sustanciales por debajo de Dios (o Trinidad de hipóstasis divinas), que, de acuerdo con nuestra filosofía, son dos: almas de distintos grados (los ángeles mismos son incluidos en este número) y el cuerpo o materia. ${ }^{5}$

Los dos tipos de sustancias son las almas, que podemos denominar sustancia inmaterial o incorpórea, y la materia. Notamos ya en esta afirmación que el primer tipo contempla distintas clases o grados en él.

Cudworth se propone en este libro enfrentarse a los diferentes ateísmos que ganan terreno en su tiempo, los cuales pueden rastrearse hasta la filosofía antigua, y que llevan a concepciones fatalistas donde se pierde la noción de libertad. Las cuatro formas que determina tienen el mismo problema: no aceptan la anterioridad, la superioridad, de la sustancia inmaterial por sobre lo material. En el mejor de los casos, los ateos crean un monstruo al proponer una materia animada por sí misma, olvidando la verdadera naturaleza de esta

Ralph Cudworth. An Interpretation, Cambridge, Cambridge University Press, 1951, [pp. 107-113].

${ }^{5}$ TIS, Prefacio, vi. 
sustancia. ${ }^{6}$ Para Cudworth, la materia ocupa el último lugar en la escala de seres, al carecer de poder vital y depender del ámbito espiritual para cualquier tipo de actividad. Así redefine el dualismo: "Porque concebimos que los principales géneros de ser deben mejor ser expresados así: extensión resistente y antitípica (antitypous), y vida (es decir, energía interna y actividad propia)". 7 Afirma que esto ya había sido sostenido en la antigüedad y expresa que es una mejor concepción, una mejoría con respecto al dualismo cartesiano.

Siguiendo a Passmore y de acuerdo con el texto recién citado, es importante entender que la dualidad no es la de pensamiento y res extensa, la de la mente que se opone al cuerpo, sino la de aquello que presenta actividad y aquello que es pasivo. ${ }^{8}$ La materia tiene como característica principal, más allá de su extensión, la imposibilidad de cualquier tipo de movimiento, de pensamiento o de vitalidad. Sin embargo, se observa la materia en movimiento y Cudworth explica que eso depende de la sustancia incorpórea, que es aquella que tiene como característica principal el poder vital, sin necesidad siquiera de tener consciencia. Esta sustancia, a diferencia de la extensa, carece de partes: "hay una sustancia distinta del cuerpo, denominada como carente de partes separables unas de otras, que puede penetrar el cuerpo, y, por último, activa por sí misma, y que tiene una energía interna distinta a la del movimiento local". ${ }^{9}$ Esa sustancia puede penetrar el cuerpo y es principio de movimiento.

El origen de todo, sin embargo, está por encima de esos dos tipos de sustancia. Cudworth presenta al Dios unitrino, origen de toda la creación e identificado con el amor intelectual, único auto-originado, bondad esencial y sustancial, y que:

$\mathrm{Al}$ tener plenitud y fecundidad infinitamente desbordantes, se da a sí mismo sin envidia, gobierna dulcemente todo, de acuerdo con la mejor sabiduría,

${ }^{6}$ TIS, Cap. III, XXX-XXXV, pp. 134-145. Strok, Natalia, "Un monstruo con cuatro cabezas que se devoran entre sí: materialismo y naturaleza plástica en Ralph Cudworth", en Diánoia, vol. 64, No 83 (noviembre de 2019-abril de 2020), DOI: https://doi.org/10.22201/iifs.18704913e.2019.83.XXXX, pp. 2-11 [1-19].

7 TIS Cap. III, XXXVII, 16, p. 159.

${ }^{8}$ Passmore, Ralph Cudworth..., pp. 19-28.

${ }^{9}$ TIS, Prefacio, xvi. Sobre la unidad de la sustancia inmaterial o alma, ver Allen, Keith, "Cudworth on Mind, Body and Plastic Nature", Philosophy Compass 8/4 (2013), p. 343 [337-347] 
sin ninguna fuerza o violencia (al estar todas las cosas sujetas naturalmente a esta autoridad, y obedeciendo verdaderamente sus leyes), y reconcilia la totalidad del mundo en armonía. ${ }^{10}$

El Dios de TIS es sabiduría, que armoniza el mundo de acuerdo con su inteligencia, que se ofrece a sí mismo en el modo de la emanación platónica. Cudworth quiere marcar también que no se trata de un Dios que intervenga en cada evento de la creación, ni que ejerza violencia sobre ella, sino que establece su ley de modo natural y lo hace a través de una herramienta específica a la que denomina naturaleza plástica (plastic nature). Explica:

Por lo cual, dado que ni todas las cosas son producidas fortuitamente o por un mecanismo sin guía de la materia, ni puede pensarse razonablemente que Dios mismo hace todas las cosas inmediata y milagrosamente, debe concluirse que hay una naturaleza plástica bajo Él, como un instrumento inferior y subordinado, que ejecuta como esclava esa parte de su providencia que consiste en el movimiento regular y ordenado de la materia, de modo tal que haya también, además de ésta, una providencia superior que debe ser reconocida, la cual, al presidir sobre todo, muchas veces suple los defectos de ella, y a veces prevalece ante ella, ya que esta naturaleza plástica no puede actuar por elección, ni con criterio. ${ }^{11}$

La naturaleza plástica es, entonces, el instrumento ciego de Dios para gobernar la materia que es absolutamente pasiva. Esta naturaleza plástica es sustancia inmaterial que trabaja internamente en la materia para darle movimiento de acuerdo con la providencia divina que ella comunica, es una especie de mensajera que incluso a veces puede equivocarse, aunque involuntariamente, para lo cual Cudworth establece una providencia superior que puede corregirla. Así, las almas que tienen algunos de los seres en este universo son superiores a aquella porque presentan algún tipo de consciencia, como veremos a continuación.

En suma, la metafísica de Cudworth contempla a un Dios, principio intelectual de todo, que imprime su sabiduría en la naturaleza, la cual gobierna a su vez la materia y ocupa el lugar más bajo en la escala de seres, pero antes

${ }^{10}$ TIS, Cap. III, XVIII, p. 117.

${ }^{11}$ TIS, Cap. III, XXXVII, 5, p. 150. 
que aquella materia, que es la última, por carecer de toda actividad. El nivel más alto es el de la vida racional de los seres animados, aunque obviamente por debajo de la perfección de la mente intelectual de Dios. Esto significa que Dios, las almas de animales y hombres, y la naturaleza plástica son todas vida activa, en oposición a la materia, que es absolutamente pasiva y depende de la vida activa en cada aspecto. El mundo se compone de materia y sustancia inmaterial, pero no de forma separada sino en conjunción, ya que la materia es absolutamente dependiente de la sustancia incorpórea y esta última no se encuentra separada de la materia, como veremos a continuación.

En cuanto a la causalidad, Cudworth sigue los lineamientos generales del llamado neoplatonismo, es decir, el único poder causal es aquel que tiene el poder suficiente para producir el efecto, y eso significa contener al efecto. Se trata de una causa eficiente que contiene igual perfección en sí que el efecto, causalmente. Nada puede ser creado a partir de la nada quiere decir que hay un único creador de lo existente, que es Dios, quien produce a partir de su propia naturaleza de forma emanativa. ${ }^{12} \mathrm{El}$ resto de los seres, imperfectos, no pueden crear nuevas sustancias sino modificaciones y accidentes sobre las sustancias creadas por Dios. ${ }^{13}$ La naturaleza plástica y las almas pueden realizar modificaciones y producir nuevos accidentes. ${ }^{14} \mathrm{La}$ naturaleza lo hace de forma necesaria y de manera inexplicable, siguiendo ciegamente el designio divino, las almas racionales son aquellas que se sustraen a esta necesidad y actúan libremente.

\section{La naturaleza inmaterial}

El universo que concibe Cudworth no contempla sólo movimiento mecánico. Para este filósofo se sigue advirtiendo finalidad y teleología, y por eso Passmore sostiene que la división esencial en su filosofía es entre aquello que es mecánico

12 TIS, Cap. V, p. 739: "And thus is it granted, that no substance could be created, or brought out of nonexistence into being, but by the sole efficiency of an absolutely perfect Being, which hath both greater perfection, (it eminently containing all things in it,) and also a sufficient emanative or creative power."

13 TEIM IV, 1, 5, p. 77.

14 TIS, Cap. III, XXXVII, 2, p. 147: "Wherefore the divine law and command, by which the things of nature are administered, must be conceived to be the real appointment of some energetic, effectual and operative cause for the production of every effect." 
y aquello que es teleológico, no entre aquello que piensa y aquello que no lo hace. ${ }^{15}$ En este sentido, el profesor de Cambridge rechaza la interpretación cartesiana de los animales como máquinas y con ello la gran distinción entre ellos y los seres humanos. ${ }^{16}$ Cudworth sostiene la teleología que muchos de sus contemporáneos están abandonando, y por eso su metafísica presenta cierta complejidad en lo concerniente a la naturaleza inmaterial.

Dentro de la sustancia inmaterial o incorpórea, denominada "vida", Cudworth realiza otra división que es la que se da entre aquello que tiene movimiento regular y que persigue un fin sin previa deliberación, que incluye movimientos naturales en general, a lo que llama "poder plástico", y aquel movimiento que es fruto de una deliberación, de lo que es ejemplo, sobre todo, la mente humana. Por eso, en este universo no hay únicamente mecanicismo, pero tampoco ocasionalismo. Explica nuestro filósofo:

Y, entonces, de nuevo, la vida o la auto actividad interna debe ser subdividida así, ya sea que actúa con consciencia o synaesthesis expresa, o ya sea sin ella; la última es la vida plástica de la naturaleza, de modo que pueda haber una acción distinta al movimiento local, o una energía vital, que no esté acompañada con esa imaginación o consciencia, que hay en las energías de la vida animal. ${ }^{17}$

Entre los tipos de movimiento, entonces, contamos el movimiento local, la energía vital inconsciente y la energía animal o consciente. La energía vital se divide de acuerdo con el origen propio o externo del comando para actuar.

\section{1. Naturaleza plástica}

Como ya adelantamos, la naturaleza plástica es el instrumento subordinado que ejecuta la providencia divina en el mundo natural, sin actuar por elección o con criterio alguno, sino ciegamente, aunque con arreglo a fines. ${ }^{18}$ De hecho, hay

\footnotetext{
${ }_{15}$ Passmore, Ralph Cudworth..., p. 24.

16 TIS, Cap. V, 846-7.

${ }^{17}$ TIS Cap. III, XXXVII, 16, p. 159.

${ }^{18}$ Cudworth enumera una cantidad de autores que sostuvieron algo similar, entre quienes se destacan sobre todo Plotino, pero también Platón y Aristóteles. Sin embargo, debe aclararse que, si bien presenta gran número de antecedentes, su
} 
una providencia superior que incluso suple los errores que ella pueda cometer, los cuales por supuesto son involuntarios. Cudworth entiende que de esta manera explica más en la filosofía natural que lo que puede explicar el mecanicismo. La ley verbal de Dios se expresa en esta causa eficiente, energética y operativa, de modo de producir cada efecto, sin su intervención directa. ${ }^{19}$ De este modo, como dijimos, la naturaleza plástica evita tanto el problema de un origen fortuito para el movimiento, como admitir la intervención de Dios en cada evento. ${ }^{20}$

Los problemas que presentan aquellos filósofos del mecanismo se resuelven, entiende Cudworth, con la incorporación de una naturaleza plástica que es la ley natural que actúa en la materia de la totalidad del universo, que mantiene la cantidad de movimiento y lo distribuye de acuerdo con las leyes naturales:

Ahora, si hay una naturaleza plástica que gobierna el movimiento de la materia en todas partes, de acuerdo con leyes, no puede darse razón de por qué la misma no podría también extenderse más allá en la disposición regular de la materia, en la formación de plantas, animales y otras cosas, de modo de proporcionar un marco coherente apto y armonía a la totalidad del universo. ${ }^{21}$

concepto de naturaleza plástica es original por lo cual consideramos que resulta desacertado interpretarlo como un autor anacrónico o poco original como supo hacerse en el siglo XX, por ejemplo, en la interpretación de Cassirer (1932): Cassirer, Ernst, Individuum und Kosmos in der Pbilosophie der Renaissance. Die Platonische Renaissance in England und die Schuel von Cambridge, Hamburg, Felix Meiner Verlag, 2002, p. 223. Ver Armour, Leslie, "Trinity, Community and Love: Cudworth's Platonism and the Idea of Love", en Hedley, Douglas y Sarah Hutton (eds.), Platonism at the Origins of Modernity, Dordrecht, Springer, 2008, p. 118 [113-129]. Sobre la fuerte influencia de Plotino, ver Lotti, Brunello, "Il mondo animato. Le fonte plotiniane del concetto de Natura Plastica in Ralph Cudworth", en Gianfranco Fiaccadori, "In Partibus Clius". Scritti in Onore Di Giovanni Pugliese Carratelli, Napoli, Vivarium, pp. 461-520.

${ }^{19}$ TIS, Cap. III, XXXVII, 2, p. 147.

${ }^{20}$ Los dos extremos: un Dios que es observador pasivo, por una parte, o que participa en cada uno de los eventos directamente, denominado "ocasionalismo", por otra.

${ }^{21}$ TIS, Cap. III, XXXVII, 5, p. 151. 
La armonía del mundo depende, entonces, de la naturaleza plástica, que actúa en toda la materia del universo, es decir, gracias a ella se produce regularidad en los seres y la conformación de una totalidad armónica. Esa naturaleza no sólo es principio del movimiento, sino también formadora del mundo material, que por supuesto incluye el mundo animal. ${ }^{22} \mathrm{El}$ profesor de Cambridge sostiene además que aceptar una naturaleza plástica evita necesitar de cualidades ocultas que actúen en la materia, algo que incorporan otros filósofos para explicar las causas de los fenómenos, ${ }^{23}$ lo que, a su entender, es aceptar directamente la ignorancia sobre las causas de las cosas. ${ }^{24}$

La naturaleza plástica "es arte en sí misma, que actúa inmediatamente sobre la materia como un principio interno". ${ }^{25} \mathrm{El}$ movimiento que se percibe en el universo, entonces, se inicia internamente en la materia por el efecto de esa naturaleza. Al ser un poder interno, Cudworth considera que su arte es superior al arte del ser humano, en tanto este es accionar externo sobre la materia. Así, la naturaleza plástica puede ser interpretada como un "alma" en lo material. ${ }^{26}$

Otra diferencia que tiene la naturaleza plástica con respecto al accionar humano es que la naturaleza no delibera ni consulta, mientras que el accionar humano tiene una previa deliberación, segundos pensamientos y búsquedas. La comparación aquí ya no es con respecto a una actividad sobre la materia, sino al propio accionar deliberativo del alma humana, aunque luego ello se opere en la materia. Así, la naturaleza es comparable al sello o a la impresión del arte omnisciente de Dios, del divino entendimiento, que es la verdadera ley de todo. En tanto sello, entonces, la naturaleza plástica no es la ley divina en sí misma, aunque la sigue directamente, y entonces no actúa por sí misma sino de modo artificial de acuerdo con la orden que le imprime la divinidad. Cudworth

\footnotetext{
${ }^{22}$ Justamente el sentido de "plástico" en este contexto es el de aquello que forma o da forma (virtud o fuerza plástica), proveniente de $\pi \lambda \alpha \sigma \tau \iota \dot{\eta}$ griego.

23 Entre los cuales se destacan los ateos hilopáticos o anaximándricos de su clasificación. Las causas ocultas en la materia era el modo de explicación del aristotelismo al que se enfrenta Cudworth.

24 TIS, Cap. III, XXXVII, 7, p. 154.

25 TIS, Cap. III, XXXVII, 8, p. 155.

26. TIS, Cap. III, XXXVII, 9, p. 156.
} 
sostiene que la naturaleza es la expresión del arte perfecto, mientras que el arte humano es imperfecto. ${ }^{27}$

Sin embargo, la naturaleza plástica, a diferencia del arte divino que es abstracto y separado, sólo se encuentra de forma concreta y encarnada. El conocimiento divino, de hecho, no puede ser incorporado a la materia como si fuera su alma, su naturaleza tan sutil y refinada sólo permite que exista en la mente de Dios. Entonces, si la ley divina es el arte arquetípico, la naturaleza es el arte ectípico (ectypal) o representativo, un sello viviente que, aunque actúe de acuerdo a ese arquetipo, no comprende ni entiende la razón por la cual actúa. ${ }^{28}$ Cudworth ejemplifica esta relación con la diferencia entre el verbo mental

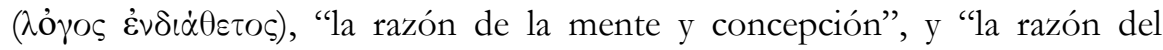

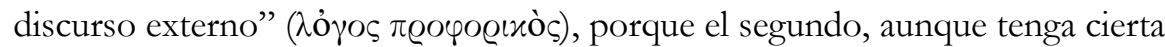
impresión del primero, en sí mismo no es más que sonido articulado, desprovisto de entendimiento. ${ }^{29}$ También compara esta relación entre arte divino y naturaleza plástica con el arquitecto y el constructor, siendo el primero el que conoce las leyes y diseña el plano, mientras que el segundo, el que materializa eso que diseñó el primero, sin entender demasiado la ciencia por la cual se hizo. ${ }^{30}$ Afirma: "La naturaleza no es maestra de ese arte consumado y sabiduría de acuerdo con la cual actúa, sino sólo sirvienta de ella, y una ejecutora esclava de sus preceptos". ${ }^{31}$ De eso se trata el estatus subordinado a Dios.

Y aunque había resaltado el arte de la naturaleza plástica como superior al humano en tanto divino, Cudworth entiende que la naturaleza encuentra el

27 TIS, Cap. III, XXXVII, 10, p. 155. Hay un error en la paginación de la edición: debería ser 157, pero retrocede a 155 . Esto repercutirá en todo el resto del libro.

${ }^{28}$ Cudworth trae la definición de arte de Aristóteles: TIS, Cap. III, XXXVII, 11, p. 155. Esta naturaleza plástica, que sigue en todo momento el designio divino, pero sin intervenir con decisiones o razonamientos, se asemeja al software de las computadoras, que trabaja de acuerdo con lo que pone en él el programador. Véase Hutton, Sarah, "Ralph Cudworth: Plastic Nature, Cognition, and the Cognizable World", en Dominik Perler y Sebastian Bender (eds.), Causation and Cognition in Early Modern Philosophy, New York, Routledge, 2020, p. 111 [105-122].

${ }^{29}$ TIS, Cap. III, XXXVII, 11, p. 155.

${ }^{30} \mathrm{El}$ ejemplo no es del todo feliz ya que en la realidad el constructor puede resolver problemas que puedan surgir en la construcción y tomar decisiones sin consultar necesariamente al arquitecto.

31 TIS, Cap. III, XXXVII, 12, p. 156. 
último lugar en la escala de seres vivificantes, encontrándose la vida del alma humana por encima de ella. Y esto ocurre justamente por esa diferencia que marcó entre el accionar humano y el arte natural, que paradójicamente es artificial: la naturaleza actúa sin conocimiento, como una esclava de la sabiduría divina, sin deliberación ni elección. Mientras que el accionar humano es libre y auto determinado, a diferencia de aquella que no sabe, sino que sólo hace, determinada por el designio divino. ${ }^{32}$

En este punto se distancia del cartesianismo porque esa sustancia incorpórea no es pensamiento consciente. Cudworth intenta explicar mejor esta situación de la naturaleza plástica a partir de ejemplos, entre los cuales se encuentra el del hábito de ciertas expresiones artísticas, como la música o la danza, y también el de los instintos animales, que me interesa en particular. Afirma:

Más aún, que algo pueda actuar de modo artificial y de acuerdo con fines, sin comprender las razones de lo que hace, puede ser evidenciado mejor a partir de los instintos naturales que existen en los animales, que sin conocimiento los dirige a actuar regularmente, tanto por su propio bien, como por el bien del universo. ${ }^{33}$

Este accionar sin cálculo contribuye a la armonía del universo en su regularidad. Los ejemplos son los clásicos: las abejas que hacen miel y construyen sus panales, las arañas que fabrican sus redes, y los pájaros que arman sus nidos, todos con gran ingenio, aunque estos animales carezcan de cualquier tipo de deliberación para su realización y lo realicen con cierta necesidad. Cudworth llega a afirmar que "estos instintos de la naturaleza en ellos (los animales) son una especie de destino en ellos". ${ }^{34}$ Sin embargo, el profesor realiza una importante afirmación con respecto a los animales que da cuenta de algo más que naturaleza plástica, porque estos, a diferencia de la naturaleza plástica, son conscientes de lo que realizan a partir de esos instintos,

32 TIS, Cap. III, XXXVII, 12, p. 156. En esta idea, Cudworth entiende no sólo estar de acuerdo con autores antiguos como Plotino, a quien cita (Enéada 2, 3, 17), sino también con contemporáneos como William Harvey.

33 TIS, Cap. III, XXXVII, 14, p. 158.

${ }^{34}$ TIS, Cap. III, XXXVII, 14, p. 158. 
aunque no comprendan sus razones. Si bien actúan de acuerdo con cierta necesidad, afirma Cudworth:

En el siguiente lugar, existe otra imperfección observable en la naturaleza plástica, que así como no comprende las razones de su propia acción, tampoco es clara y expresamente consciente de lo que hace, en cuyo respecto no sólo es inferior al arte humano, sino también que esa manera de actuar que hay en los propios (animales) brutos, quienes aunque no entienden las razones de esas acciones, que su instinto natural los conduce a realizar, de todos modos generalmente se concibe que son conscientes de ellas, y las hacen con imaginación, mientras que la naturaleza plástica parece no tener imaginación animal en la formación de plantas y animales, ni

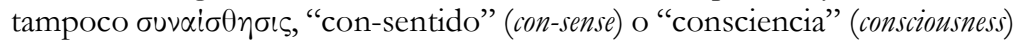
expresa de lo que hace..$^{35}$

Es decir, la naturaleza plástica no sólo actúa de modo fatal, sin saber las razones por las cuales realiza sus actos, sino que además resulta absolutamente carente de cualquier tipo de consciencia. Los animales, por su parte, si bien no conocen las razones de sus actos, que en tanto instintivos tienen también el modo de la fatalidad, alcanzan, sin embargo, algún tipo de consciencia en su accionar, aunque sólo al nivel de la imaginación. De este modo, el autor diferencia el alma de los animales de la naturaleza plástica.

En contra de quienes sólo pueden concebir una sustancia inmaterial consciente o pensante, como la res cogitans, Cudworth presenta también los ejemplos de vida inconsciente en los seres humanos: sueño profundo, letargos, apoplejía, embriones en el vientre materno son casos de vida sin consciencia. Esto muestra que no es necesario que la energía vital deba ser también consciente. Afirma: "Ahora, si las almas de hombres y animales están en algún momento sin consciencia y auto percepción, entonces debe admitirse con necesidad que la consciencia clara y expresa no es esencial para la vida". ${ }^{36}$ Las almas, entonces, sustancia inmaterial del universo al igual que la naturaleza plástica, pueden también actuar de modo inconsciente. Otros ejemplos de esto los encuentra en los casos del matemático dormido, que sigue utilizando sus conocimientos en resoluciones de problemas en sueños, o el músico

35 TIS, Cap. III, XXXVII, 15, pp. 158-9.

36 TIS, Cap. III, XXXVII, 17, p. 160. 
adormilado que sigue tocando su música. Así se entiende que las almas presenten rasgos inconscientes incluso en lo más cotidiano, que es la unión al cuerpo. Así lo expresa el autor:

Esa empatía vital por la cual nuestra alma está unidad y sujeta, como si fuera en un nudo, al cuerpo es una cosa que tenemos sin consciencia directa, sino sólo en sus efectos. Tampoco podemos decir cómo llegamos a ser tan diferentemente afectados en nuestras almas a partir de los tantos diversos movimientos que se realizan en nuestros cuerpos. ${ }^{37}$

Una vez más, Cudworth se distancia del dualismo cartesiano en la relación que propone para sus dos sustancias, ya que sostiene que aquello que produce el movimiento de la materia lo hace a partir de cierta empatía vital con la materia. Así como en la unión de alma y cuerpo, también la naturaleza inmaterial, que actúa de acuerdo con fines, pero sin consciencia, tiene afinidad con aquello sobre lo que actúa. En un caso y en otro, Cudworth sostiene la empatía entre las distintas sustancias, fundada en la sabiduría divina, al punto de no encontrarse estas dos sustancias de modo separado.

Sobre el tema de la ausencia de consciencia, el profesor de Cambridge sostiene que en las almas también se encuentra un "poder plástico interior" que es formador de pensamientos, aunque sin consciencia de ello. Y más evidente aún se ve ese movimiento vital en casos como, por ejemplo, el de la respiración o la circulación sanguínea. Concluye:

Ahora, así como no tenemos en absoluto poder voluntario sobre la sístole y la diástole del corazón, tampoco somos conscientes en nosotros mismos de alguna energía de nuestra alma que los cause, y por eso podemos razonablemente concluir a partir de esto, que existe cierta energía vital, desprovista de imaginación animal o consciencia expresa y auto percepción. ${ }^{38}$

Los movimientos involuntarios y regulares en la propia naturaleza de animales irracionales y racionales dan cuenta de la existencia de un poder vital

${ }^{37}$ TIS, Cap. III, XXXVII, 17, pp. 160-1.

38 TIS, Cap. III, XXXVII, 17, p. 161. 
inconsciente, carente de pensamiento, imaginación o percepción: poder plástico.

La pregunta que surge inmediatamente es cómo se produce el funcionamiento de esta naturaleza, así caracterizada. Cudworth afirma: "Ahora, lo que actúa no por algún conocimiento o imaginación, voluntad o apetito propio, sino sólo de modo fatal de acuerdo con leyes e impresiones hechas en esto (pero de modos diversos en diferentes casos) puede ser dicho también que actúa mágica y empáticamente". ${ }^{39}$ Entonces, la relación se produce fundada en la empatía y el accionar es mágico. La apelación a lo mágico aquí parece incorporarse para mostrar la contraposición con un accionar mecánico, por una parte, y como aquello que no se explica y que no se produce a partir de una deliberación propia, por otra. Se aprecia que esa magia, en todo caso, se funda en la inteligencia divina porque lejos está de ser caótica; ${ }^{40}$ todo lo contrario: se trata de la ley de la naturaleza, ${ }^{41}$ que nada tiene que ver con el mecanicismo de la época. Entonces, la unión entre alma y cuerpo, entre sustancia activa y pasiva, se produce empática y mágicamente, motivo que Cudworth encuentra ya presente en Alejandro de Afrodisia, ${ }^{42}$ dejando claro que sus antecedentes no sólo se encuentran en la tradición platónica, especialmente en Plotino, sino también en la aristotélica.

Resulta interesante que el profesor de Cambridge repase la posición de Aristóteles con respecto a la naturaleza plástica y considere también la posibilidad de proponer una naturaleza plástica general que gobierne la totalidad y naturalezas plásticas particulares en cada ente. Sin embargo, esto no parece más que una hipótesis para conformar a aquellos que no aceptarían que se tratara de una única naturaleza plástica que gobierna en todo el universo, siguiendo ciegamente la ley divina. ${ }^{43}$

39 TIS, Cap. III, XXXVII, 18, p. 162.

40 Sobre el concepto de "magia" en los Platónicos de Cambridge y la época, ver Copenhaver, B.P., Magic in the Western Culture. From Antiquity to the Enlightenment, Cambridge, Cambridge University Press, 2015, pp. 398-405.

${ }^{41}$ Se trata de una ley metafísica y no física. Véase Lotti, “Il mondo animato”, p. 504.

${ }^{42}$ Las citas que ofrece como apoyo son del De Fato de Alejandro de Afrodisia.

${ }^{43}$ Ver TIS, Cap. III, XXXVII, 25, p. 176: “And this perhaps may we the minds of those, who cannot but think it too much to impose all upon one plastic nature of the universe". Cudworth plantea su dualismo conformado por dos sustancias, aunque una de ellas pueda diferenciarse de acuerdo con la característica de la consciencia o la 


\subsection{Alma}

Hemos visto que el poder plástico actúa en la totalidad de la naturaleza, incluso en aquellos seres que tienen alma. Entonces, para exponer el tema de esta sección es necesario caracterizar en los entes animados, irracionales y racionales, aquello que es propiamente su alma.

En primer lugar, las almas son aquellas sustancias inmateriales que presentan algún tipo de consciencia. Los animales y los seres humanos y angélicos tienen alma. Esas almas están unidas a sus cuerpos al mismo modo que la naturaleza plástica se une a la materia. Esta característica de la unión, entonces, no es propia de las almas sino de toda la sustancia inmaterial en el universo de Cudworth, al punto de que no existen las almas sin cuerpos. ${ }^{44} \mathrm{El}$ profesor de Cambridge plantea pensar a cada alma como toda en todas las partes de su cuerpo en tanto el alma es una sustancia inextensa y sin partes, ${ }^{45}$ pero sobre todo como unida siempre a algún tipo de cuerpo. ${ }^{46}$

Entonces, podemos sostener que lo propio del alma es su capacidad de ser consciente. La consciencia es, para nuestro autor: "esa duplicación que se

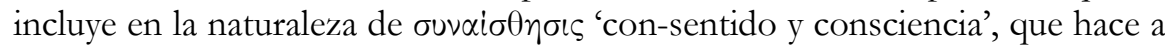
un ser presente a sí mismo, atento a sus propias acciones u opuesto a ellas, que

inconsciencia. Sin embargo, nada en su explicación avalaría pensar en distintas sustancias inmateriales sino en una única, y así en una única naturaleza plástica. Veremos qué pasa con las almas en la siguiente sección.

${ }^{44}$ TIS, Prefacio, p. xvi: "souls are always united to some body or other, as also, that all rational and intellectual creatures consist of soul and body".

45 TIS, Cap. V, p. 782: "We say it is contradictious to the nature of that substance,

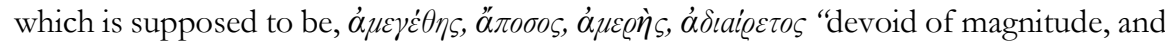
of quantity, and of parts indistant, and indivisible"; that it should be otherwise united to, or conjoined with an extended body, than after this way, which is looked upon as such conjuring; namely, that the whole of it should be present with, and act upon every part thereof'. Cudworth explica que esta doctrina es bien presentada por Plotino.

46 TIS, cap. V, pp. 794 y ss. Cudworth muestra la coincidencia entre la cábala pitagórica que sostiene la unión permanente del alma a algún tipo de cuerpo con la doctrina cristiana que requiere de un cuerpo para la resurrección, aunque no un cuerpo terrestre sino etéreo. Esta interpretación que realiza Cudworth habilita la posibilidad de ubicar el principio de individuación del alma en la unión con un cuerpo, que la acompaña incluso después de la muerte. 
se percibe a sí mismo en el hacer o el sufrir y que tiene una fructificación o disfrute de sí mismo. ${ }^{47}$

Cudworth explica que en el alma se produce una duplicación que es la consciencia, donde aquella se encuentra consigo misma, se hace presente a sí misma y por eso se vuelve atenta a su propio accionar. En ese accionar puede darse tanto el placer como el sufrimiento. Esto es algo que se encuentra ya presente en los animales que actúan bajo la influencia de los instintos y sólo alcanzan el nivel de la imaginación, más no así el de la razón. Y lo que primero debemos advertir es que esta consciencia es auto-consciencia.

En este punto conviene que repasemos los distintos niveles de conocimiento que contempla el autor. En TEIM Cudworth presenta su gnoseología. Una vez más discute con ciertas filosofías de su época, que se atienen sólo a lo material, y para eso explica cómo se produce el conocimiento. En primer lugar, va a subrayar la relación con la divinidad que funda todo conocer:

Ahora, todo el conocimiento y sabiduría que hay en las creaturas, ya sean ángeles u hombres, no es nada más que la participación de esa sabiduría de Dios eterna, inmutable e increada, o varias signaturas de ese único sello arquetípico, o como tantos múltiples reflejos de un único y mismo rostro, hechos en muchos cristales, del cual algunos son más claros, otros más oscuros, algunos que permanecen más cercanos, otros más lejanos. ${ }^{48}$

El conocimiento, de acuerdo con la tradición platónica, se da por participación en la sabiduría divina. Y este se produce de acuerdo con el modo en que los participados reciben esa luz de Dios. ${ }^{49}$ En su discusión con las corrientes que considera ateas, Cudworth explica que el conocimiento se produce en el alma y que, en rigor, sólo se considera como tal aquel que

47 TIS, Cap. III, XXXVII, 16, p. 159.

${ }^{48}$ TEIM, libro I, cap. 3, 7, p. 26.

${ }^{49}$ Motivo platónico que puede encontrarse, por ejemplo, en Nicolás de Cusa: Idiota. De Mente (1450). 
proviene del vigor interno de esta, es decir, los sentidos no aportan verdadero conocimiento. ${ }^{50}$ Sostiene:

Por lo cual, el resultado correcto y genuino de esta antigua filosofía atomista, que es triunfo de la razón sobre la sensación, no es otro que este: que el sentido solo no es el criterio o el juez de lo que existe real y absolutamente fuera de nosotros, sino que hay una facultad intelectual más alta y superior en nosotros que juzga a nuestros sentidos, que descubre lo que es falaz y fantasioso en ellos, y declara qué es y qué no es absolutamente. ${ }^{51}$

Cudworth pone siempre el acento en la actividad interna de la mente porque, incluso en la información proveniente de los sentidos, el alma trabaja de forma activa. Explica: "La sensación no es sino el ofrecimiento o la presentación de algún objeto a la mente para darle una ocasión de ejercitar su propia actividad interna sobre él". ${ }^{52} \mathrm{Y}$ por ello se entiende que el verdadero conocimiento sea aquel que no utiliza nada externo a la propia alma:

De modo que el conocimiento no es una pasión que provenga de nada fuera de la mente, sino un esfuerzo activo de la fuerza, el vigor y el poder interno de la mente, al desplegarse a sí misma desde el interior, y las formas inteligibles por las que las cosas son entendidas o conocidas no son sellos o impresiones pasivamente impresas en el alma desde fuera, sino ideas que se extienden vitalmente o se ejercen activamente desde el interior. ${ }^{53}$

A diferencia de la naturaleza plástica que actuaba sobre la materia como el sello divino directamente, la mente contiene en sí las ideas que reflejan la sabiduría divina como una "omniformidad", es decir, tiene todas las formas a partir de las cuales puede ejecutar su actividad y extenderse. ${ }^{54} \mathrm{Si}$ bien la naturaleza plástica era actividad, había algo estático en su recepción de la información a partir de la cual actuaba, porque ella no reflexiona sobre esta, ni

${ }^{50}$ Hedley lo denomina idealismo epistemológico. Véase Hedley, Douglas, "Ralph Cudworth as Interpreter of Plotinus", en Stephen Gersh (ed.), Plotinus' Legacy, Cambridge, Cambridge University Press, 2019, p. 147, (146-159).

${ }^{51}$ TEIM, Libro II, cap. 6, 3, p. 47.

52 TEIM, Libro III, cap. 3, 2, pp. 57-8.

${ }^{53}$ TEIM, libro IV, cap. 1, 1, pp. 73-4.

${ }^{54}$ TEIM, Libro IV, cap. 1, 5, p. 77. 
realiza ningún tipo de razonamiento, sino que sólo la ejecuta. En contraposición, la naturaleza humana alcanza un verdadero conocimiento en el accionar del alma sobre sí misma, a lo que se denomina mente, que es una auto ejercitación, actuando a partir de su propia actividad, es decir, auto determinándose. ${ }^{55}$ Por su parte, los animales no tienen exactamente este tipo de actividad mental, sino más bien un punto medio entre la expresión de la naturaleza y la reduplicacion de la mente.

Como adelantamos en la sección anterior, los animales cuentan con percepción sensitiva e imaginación, a diferencia de la naturaleza plástica que carecía incluso de esto. Para el profesor de Cambridge sensación e imaginación son casi idénticas porque es aquello producido en el alma a partir del contacto con el cuerpo, ya sea en presencia o no de eso sentido o imaginado, y donde el alma presenta cierta pasividad. ${ }^{56}$ En contraposición, la capacidad de juzgar sobre aquellos sentidos es propia de la naturaleza humana. Afirma Cudworth:

¿qué razón podría darse de por qué los animales brutos que tienen los mismos sentidos que los hombres, incluso algunos de ellos más agudos, no deberían tener intelección también, y ser tan capaces de lógica, matemática y metafísica, y tener las mismas nociones de moralidad, de una deidad y de la religión como los hombres? Por eso, debe necesariamente ser garantizado que, además de las pasiones de las cosas corpóreas, o la percepción pasiva del sentido, hay en el alma de los hombres otro principio más activo o vis cognoscendarum rerum innata, "un poder cognoscitivo innato", por el cual ellos son capaces de entender o juzgar lo que se recibe desde el exterior a través de la sensación. ${ }^{57}$

Ambos seres cuentan con almas perceptivas pero las de los seres humanos alcanzar un nivel de conocimiento superior en tanto participan en

\footnotetext{
55 Carter explica que en los manuscritos no editados se encuentra el desarrollo del tema de una providencia móvil (Moveable Providence) que abre a la libertad en la naturaleza humana, en contraposición a la providencia fija y determinante a partir de la que actúa la naturaleza plástica (Carter, The Little Commonwealth of Man, pp. 90-91).

56 TEIM, libro III, cap. 4. En este capítulo, Cudworth enfatiza que es el poder racional el que determina cuándo se trata de una percepción de algo real y cuándo una fantasía. En última instancia, ambos tipos de percepciones son acompañadas por fantasías. 57 TEIM, Libro IV, cap. 1, 3, p. 75.
} 
mayor medida de la sabiduría divina y tienen la capacidad de juzgar lo que provee el sentido. Cudworth utiliza el ejemplo de la música para mostrar esta diferencia:

Dejemos a un hombre y a una bestia al mismo tiempo escuchar las mismas melodías musicales, la bestia solo apreciará ruidos y sonidos. Pero el hombre también percibirá armonía en ello, y se deleitará mucho con ella; más aún, será transportado entusiastamente por ella. Por lo cual, al percibir la bestia todos los sonidos tanto como el hombre, pero nada de la armonía, la diferencia debe surgir necesariamente de algún principio activo interno o anticipación en el hombre que la bestia no tiene. ${ }^{58}$

Así marca el autor, entonces, una diferencia en las capacidades cognoscitivas de los seres animados. A lo recibido por vía de la sensación, la naturaleza humana agrega algo propio de su alma que los animales no realizan y por eso tienen un grado más alto de actividad en ella. Esto indica su mayor cercanía a la divinidad, un grado superior de vida.

Esta distinción tiene su correlato también en el aspecto consciente del alma, porque ya afirmamos que los animales son conscientes de su accionar que tiene en la base a los instintos y la percepción sensible e imaginativa. Los seres humanos, por su parte, contarán además con la consciencia que podrá acompañar al conocimiento racional, que los animales no poseen. ${ }^{59}$ En este sentido, ambos tipos de consciencia son la duplicación que el alma realiza al desarrollar las capacidades pertinentes para cada caso, que implica la atención sobre aquello que está realizándose, y evidentemente serán distintos tipos en cuanto a los objetos de conocimiento a los que corresponden. El animal es consciente, pero sobre cierta pasividad receptiva de sensaciones e información proveniente de los instintos. La naturaleza humana ejerce sobre eso recibido por la sensibilidad, e incluso sobre ciertos rasgos instintivos, una actividad propia del carácter racional. Agregamos a este mapa del alma que, además, por

${ }^{58}$ TEIM, libro IV, cap. 2, 14, p. 95.

${ }^{59} \mathrm{La}$ consciencia para el conocimiento racional es supuesta por Cudworth en su obra. Puede verse por ejemplo TEIM, libro IV, cap. 1, 3 . 
los ejemplos antes presentados, ella puede trabajar también de modo inconsciente, a partir del hábito. ${ }^{60}$

Pécharman aclara que por debajo de la auto consciencia también existe una consciencia a secas, que no implica una duplicación y es patente, por ejemplo, cuando el alma distingue entre sensaciones y fantasías. Además, determina un nivel más alto de consciencia en la naturaleza humana, que es aquella de la personalidad ( $I$ my self), distinta a la autoconsciencia de la actividad racional y sensible-imaginativa. Este nivel más alto es el que permite la autodeterminación, dando lugar al sujeto de responsabilidad moral. Carter agrega, a partir de los manuscritos aún no publicados, que este poder de autokinesis (auto-movimiento) es un don de Dios dado a la naturaleza humana por su gracia. ${ }^{61}$

En el TFW Cudworth explica la constitución de la personalidad a partir de la capacidad del alma de comprenderse como un todo, en una reduplicación:

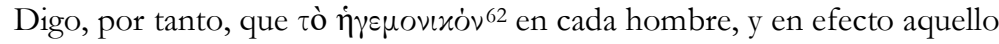
que propiamente somos nosotros mismos, (preferimos considerar que tenemos y no que somos esas otras cosas de naturaleza necesaria), es el alma que se comprehende a sí misma: todos sus asuntos e intereses, sus habilidades y capacidades, y se sostiene, como si fuera en su propia mano, como si estuviera reduplicada sobre sí misma; que tiene un poder de intencionar o ejercerse a sí misma en mayor o menor medida, en la consideración y la deliberación, en resistirse a los apetitos bajos que se le oponen, tanto en utilidad, como en razón y en honestidad. ${ }^{63}$

Esto hegemónico es el centro del alma, en el que la unidad permite la personalidad en cada individuo, muestra la actividad más comprehensiva del

\footnotetext{
${ }^{60}$ Me refiero a los ejemplos de matemáticos y músicos dormidos de la sección anterior. Sobre el hábito en el accionar humano inconsciente, ver Carter, The Little Commonwealth..., pp. 97-98.

${ }^{61}$ Pécharman, Martine, "Cudworth on Self-Consciousness and the I Myself", Vivarium 52, 2014, pp. 292-301 (287-314). Carter, The Little Commonwealth..., pp. 99-100.

62 "Lo hegemónico", término que Cudworth toma del estoicismo. Sobre Cudworth y el estoicismo: Sellars, John. "Stoics against Stoics in Cudworth's A Treatise of Freewill." British Journal for the History of Philosophy 20:5, 2012, pp. 935-952.

${ }^{63} \mathrm{TFW}$, cap. X, p. 178.
} 
alma, y así la libertad con respecto a la necesidad natural. ${ }^{64}$ Se trata de una segunda duplicación, sobre la duplicación que ya ejercía la autoconsciencia.

Ahora bien, habiendo mostrado la capacidad consciente en animales y seres humanos, retomemos aquello que tiene cierta fatalidad en los seres animados, donde se hace difuso el límite con la naturaleza plástica, a lo que Cudworth se refiere como poder plástico. Hemos ya dado el ejemplo de la sístole y la diástole, pero podemos agregar infinitos más como el de la respiración y el funcionamiento de los órganos del cuerpo. Hay algo natural en el cuerpo de animales inferiores y superiores, que además de ser inconsciente, resulta totalmente involuntario. Explica en TEIM:

De nuevo, el alma es a veces tan afectada por movimientos comunicados desde los nervios que pertenecen al estómago y aesophagus, "esófago", como para percibir o enterarse del hambre o la sed en esas partes del cuerpo, y algo parecido puede decirse del resto de los dolores o placeres, lascivias y agitaciones del cuerpo, que el alma percibe como cosas existentes en alguna parte específica del propio cuerpo, cuando los nervios son movidos de esa determinada manera. ${ }^{65}$

Hay cierto mecanismo en los cuerpos de los seres animados que parece responder al accionar ciego y providencial de la naturaleza plástica. De hecho, la formación de esos distintos órganos responde a esa legalidad divina, aunque en la formación de esos seres se acompañe con un alma particular. La muerte de los seres animados es natural, y entonces podemos decir que tiene algo de providencial, aunque en muchos casos los seres racionales puedan modificar el momento en que ello suceda de acuerdo con cálculos que les son propios. ${ }^{66}$

${ }^{64}$ Pécharman, "Cudworth on Self-Consciousness...”, p. 308-313, Breteau, Jean-Louis, "'Un grand espace pour la liberté?': Le dilemme du libre arbitre dans la pensée de Ralph Cudworth", Archives de Pbilosophie 58:3, 1995, pp. 437-41 [421-441]; Hutton, Sarah, "Salving the phenomena of mind: energy, hegemonikon, and sympathy in Cudworth", British Journal for the History of Philosophy 25:3, 2017, pp. 479-483 [465-486], y Zarka, Charles Yves, "Penser l'action libre: Cudworth contre Hobbes", en Charles Yves Zarka (ed.), Liberté et necessité chez. Hobbes et ses contemporains, Paris, Vrin, 2012, pp.72-3 [67-79]. ${ }^{65}$ TEIM, Libro III, cap. 1, 5, p. 52.

${ }^{66}$ Me refiero a someterse a tratamientos médicos para la cura de enfermedades, incluso a evitar realizar o no algunas actividades que pondrían en riesgo la vida. Los instintos 
Los animales, en quienes funcionan los instintos, presentan como peculiaridad la autoconsciencia de su actividad. Pero podemos decir que lo propio del alma es el ejercicio de esos sentidos e imaginación, antes que lo mecánico en sus cuerpos.

Una caracterización del alma y del poder plástico se encuentra en TEIM, donde podemos marcar la diferencia y la similitud entre una y otra:

Aunque ellos (los intelectos) no tienen las ideas actuales de todas las cosas, mucho menos están las imágenes o esculturas de todas las diversas especies de cosas existentes, fijadas y grabadas de una manera muerta sobre ellos, de todos modos las tienen a todas virtual y potencialmente comprehendidas en ese vis cognitrix, "poder cognoscitivo", del alma, que es una omniformidad potencial a través de la cual es capaz, cuando la ocasión dispone y los objetos exteriores invitan, de desenvolverse gradual y sucesivamente y desplegarse en una manera vital, al enmarcar ideas o concepciones inteligibles dentro de sí misma de cualquier cosa que tenga alguna entidad o cognoscibilidad. Así como el poder espermático o plástico contiene virtualmente en sí mismo las formas de todas las diversas partes orgánicas de los animales, y las despliega gradual y sucesivamente, enmarcando un ojo aquí y una oreja allá. ${ }^{67}$

Aquí el despliegue de lo intelectual en el alma, que forma ideas, pensamientos, que utiliza ese poder cognoscitivo que le aporta la virtualidad de las ideas de todo. Y también el despliegue del poder plástico, que contiene en sí virtualmente las ideas de todas las formas, y que va desarrollando su poder en la materia de modo de ir formando esas distintas partes. Podremos llamar a todo esto alma, pero no hay ninguna diferencia entre este poder plástico del alma y la naturaleza plástica, que es justamente formadora de materia. En este sentido decimos junto a Allen que toda la sustancia incorpórea tiene el poder

en los animales no presentan ese tipo de cálculo, aunque Cudworth admita una posibilidad de libertad de elección en los animales de modo contingente. Ver TFW, cap. XV, pp. 191-3. Sobre la evolución de este tema en los manuscritos ver Leisinger, Matthew, "Animals, Freewill, and Animal Freewill: a Development in Cudworth's Freewill Manuscripts", en https://cprg.hypotheses.org/830, 2019. Sobre la libertad moral en el alma, ver Leisinger, Matthew "Cudworth on Freewill", Philosophers' Imprint, 2020 (en prensa).

${ }^{67}$ TEIM, Libro IV, cap. 1, 5, p. 77 
de mover la materia, pero algunos seres también tienen el poder del pensamiento. ${ }^{6}$

Ese pensamiento puede torcer de alguna manera la naturalidad de la providencia divina y eso explicaría que en el universo armónico que plantea Cudworth, fundado en la sabiduría y bondad divina, se produzcan guerras y todo tipo de crueldades. Sin embargo, como la mente es reflejo de la sabiduría divina, contiene en sí ideas de moralidad ${ }^{69}$ y participa de la única mente divina, ${ }^{70}$ el gobierno de Dios está garantizado, siempre y cuando se sigan las leyes de la justicia natural.

En la última sección del TIS se encuentra una referencia a la soberanía civil, donde Cudworth critica a los ateos que consideran que la justicia y la organización política son artificiales, ya que naturalmente sólo prevalece el egoísmo, que lleva a la guerra de todos contra todos, realizando una clara referencia a Hobbes. ${ }^{71}$ Para Cudworth existe una justicia natural y por eso afirma: "la primera obligación original no proviene de la voluntad sino de la naturaleza". ${ }^{72}$ En su opinión no puede haber inconsistencia entre la religión y la soberanía civil y por eso explica:

Porque consciencia es también, en sí misma, no de una naturaleza privada o parcial, sino de una pública y común, que respeta leyes divinas, la justicia imparcial y la equidad, y el bien de la totalidad, que choca con nuestro bien egoísta y la utilidad privada. Esta es la única cosa que puede naturalmente asociar a la humanidad toda junta, permite una fundación para los cuerpos políticos y aleja esas voluntad y juicio privados, de acuerdo con el apetito y utilidad de los hombres, que es inconsistente con la misma. ${ }^{73}$

${ }^{68}$ Allen, "Cudworth on Mind ...", p. 342.

${ }^{69}$ TEIM, Libro IV, cap. 2, 14.

70 TIS, Cap. V, p. 750.

${ }^{71}$ Cita el De Cive de Hobbes: TIS, Cap. V, pp. 891 y ss.

72 TIS, Cap. V, p. 896.

73 TIS, Cap. V, p. 898. Allí Cudworth explica que esto está de acuerdo con la opinión de Platón en Leyes, libro 9. 
Para el profesor de Cambridge lo natural es actuar como una comunidad que respeta las leyes divinas que conducen la totalidad hacia el bien. ${ }^{74} \mathrm{Y}$ en este punto el autor parece querer subrayar la importancia de entender la unidad que liga todo, aunque las verdades se encuentren en cada alma y es una búsqueda interior, eso es compartido y debería poder ser notado. Esa unidad que da la divinidad a su creación se encuentra en la naturaleza incorpórea, a la que constituye no sólo con bondad sino también con amor. ${ }^{75}$

\section{Conclusión}

El universo que plantea Cudworth en su obra publicada y sus manuscritos puede ser considerado dualista. Allí se presentan dos sustancias: la corpórea o material que es extensión sin vida ni percepción alguna, y la sustancial vital, inmaterial, incorpórea. Ambas fueron creadas por una Deidad superior. La materia es absolutamente dependiente de la potencia vital que existe en la sustancia inmaterial. Por su parte, la sustancia inmaterial no existe separada de la materia. Ella actúa internamente en la materia o se encuentra unida a ella de modo de formar un ente animado.

La armonía física del mundo depende del poder plástico de la naturaleza, que también se encuentra en las almas de los seres animados. Las almas, por su parte, se caracterizan por su posibilidad de consciencia, percepción y conocimiento, que adquiere una diversidad de grados en el pensamiento de este autor. Sin embargo, el poder plástico no cesa de trabajar en los cuerpos de aquellas almas, porque es lo requerido para la vida, y así la respiración, la circulación de la sangre, las alarmas corporales como el hambre o la sed, y muchos ejemplos más, dependen totalmente de esa plasticidad en la materia.

Con esto quise mostrar la primacía de la sustancia inmaterial en Cudworth, indiscernible de la materia, y que da unidad a todo lo creado, aunque adquiere particularidad de acuerdo con el ser habitado, formando duplicaciones sobre sí misma, al punto de realizar doblemente esa duplicación y conformar

\footnotetext{
${ }^{74}$ Sobre estos temas en los manuscritos y obras de juventud de Cudworth, ver Carter, The Little Commonwealth..., pp. 109-116 y sobre su teoría política pp. 124-129.

${ }^{75}$ Sobre los tipos de gracia y el amor en el universo de Cudworth, ver Leech, David "Cudworth on superintellectual instinct as inclination to the good", British Journal for the History of Philosophy, 2017, DOI: 10.1080/09608788.2017.1330188, pp. 4-10 [1-18].
} 
la personalidad de los seres humanos auto determinados. En el universo armónico creado por Dios, todo esto se da en continuidad, y en tanto el poder cognoscitivo contiene las ideas de todas las cosas virtualmente, como un espejo en el que se refleja la sabiduría divina, podemos decir que la personalidad del ser humano es el punto de conexión de toda esa armonía natural en el plano consciente. A nivel político esto debería tener importancia, porque de esta manera la humanidad no sólo es responsable de sí misma sino también de todo el resto de la creación en un camino de reconocimiento de la unidad del todo, que reconduce hacia la divinidad. Entonces, las garantías de la unidad y armonía en el universo creado se encuentran en la sustancia inmaterial, que traduce el mensaje divino, que refleja la sabiduría de acuerdo con su capacidad y que puede extenderla a su manera, aunque el fundamento último se encuentra en una divinidad sabia, buena y poderosa.

\section{Referencias bibliográficas}

- Allen, Keith, "Cudworth on Mind, Body and Plastic Nature", Philosophy Compass 8/4 (2013), pp. 337-347.

- Armour, Leslie, "Trinity, Community and Love: Cudworth's Platonism and the Idea of Love", en Hedley, Douglas y Sarah Hutton (eds.) Platonism at the Origins of Modernity, Dordrecht, Springer, 2008, pp. 113-129.

- Breteau, Jean-Louis, “'Un grand espace pour la liberté?’: le dilemme du libre arbitre dans la pensée de Ralph Cudworth", Archives de Philosophie 58:3, 1995, pp. 421-441.

- Burden, Mark, "Ralph Cudworth's Freewill Manuscripts: A Roadmap to Dating and Analysis", en https://cprg.hypotheses.org/815, 2019.

- Carter, Benjamin, "The Standing of Ralph Cudworth As a Philosopher", en G.A.J. Rogers, Tom Sorell y Jill Kraye (eds.), Insiders and outsiders in seventeenthcentury philosophy, New York, Taylor \& Francis, 2010, pp. 99-121.

- Carter, Benjamin, The Little Commonwealth of Man: The Trinitarian Origins of the Ethical and Political Philosophy of Ralph Cudworth, Leuven, Peeters, 2011. 
- Cassirer, Ernst, Individuum und Kosmos in der Philosophie der Renaissance. Die Platonische Renaissance in England und die Schuel von Cambridge, Hamburg, Felix Meiner Verlag, 2002.

- Copenhaver, B.P., Magic in the Western Culture. From Antiquity to the Enlightenment, Cambridge, Cambridge University Press, 2015.

- Cudworth, Ralph, The True Intellectual System of the Universe, London, Richard Royston, 1678.

- Cudworth, Ralph, A Treatise Concerning Eternal and Immutable Morality: With $A$ Treatise of Freewill, editado por Sarah Hutton, Cambridge, Cambridge University, 1996.

- Hedley, Douglas, "Ralph Cudworth as Interpreter of Plotinus", en Stephen Gersh (ed.), Plotinus' Legacy, Cambridge, Cambridge University Press, 2019, pp. 146-159.

- Hutton, Sarah, "Ralph Cudworth: Plastic Nature, Cognition, and the Cognizable World", en Dominik Perler y Sebastian Bender (eds.), Causation and Cognition in Early Modern Philosophy, New York, Routledge, 2020, pp. 105-122.

- Hutton, Sarah, "Salving the phenomena of mind: energy, hegemonikon, and sympathy in Cudworth", British Journal for the History of Philosophy 25:3, 2017, pp. 465-486.

- Leech, David, "Cudworth on superintellectual instinct as inclination to the good", British Journal for the History of Philosophy, 2017, DOI: 10.1080/09608788.2017.1330188, pp. 1-18.

- Leisinger, Matthew, “Animals, Freewill, and Animal Freewill: a Development in Cudworth's Freewill Manuscripts", en https://cprg.hypotheses.org/830, 2019.

- Leisinger, Matthew, “Cudworth on Freewill”, Philosophers' Imprint, 2020 (en prensa).

- Lotti, Brunello, "Il mondo animato. Le fonte plotiniane del concetto de Natura Plastica in Ralph Cudworth", en Gianfranco Fiaccadori, "In Partibus Clius". Scritti in Onore Di Giovanni Pugliese Carratelli, Napoli, Vivarium, 2006, pp. 461-520. 
- Pécharman, Martine, "Cudworth on Self-Consciousness and the I Myself", Vivarium 52, 2014, pp. 287-314.

- Passmore, J. A., Ralph Cudworth. An Interpretation, Cambridge, Cambridge University Press, 1951.

- Sellars, John, "Stoics against Stoics in Cudworth's A Treatise of Freewill," British Journal for the History of Philosophy 20:5, 2012, pp. 935-952.

- Strok, Natalia, "Un monstruo con cuatro cabezas que se devoran entre sí: materialismo y naturaleza plástica en Ralph Cudworth", en Diánoia, vol. 64, N 83 , 2019/2020, DOI: https://doi.org/10.22201/iifs.18704913e.2019.83.XXXX, pp. 1-19.

- Strok, Natalia, "El enigma de Ralph Cudworth en la historia de la filosofía", en Revista Anales del Seminario de Historia de la Filosofía 35 (2), 2018, pp. 357-373.

- Zarka, Charles Yves, "Penser l'action libre: Cudworth contre Hobbes", en Charles Yves Zarka (ed.), Liberté et Necessité chez. Hobbes et ses contemporains, Paris, Vrin, 2012, pp. 67-79.

Recibido: 08/2020; aceptado: 12/2020 